\title{
FDR3 - A Modern Refinement Checker for CSP
}

\author{
Thomas Gibson-Robinson, Philip Armstrong, Alexandre Boulgakov, \\ and Andrew W. Roscoe \\ Department of Computer Science, University of Oxford \\ Wolfson Building, Parks Road, Oxford, OX1 3QD, UK \\ \{thomas.gibson-robinson, philip.armstrong, \\ alexandre.boulgakov, bill.roscoe\}@cs.ox.ac.uk
}

\begin{abstract}
FDR3 is a complete rewrite of the CSP refinement checker FDR2, incorporating a significant number of enhancements. In this paper we describe the operation of FDR3 at a high level and then give a detailed description of several of its more important innovations. This includes the new multi-core refinement-checking algorithm that is able to achieve a near linear speed up as the number of cores increase. Further, we describe the new algorithm that FDR3 uses to construct its internal representation of CSP processes - this algorithm is more efficient than FDR2's, and is able to compile a large class of CSP processes to more efficient internal representations. We also present experimental results that compare FDR3 to related tools, which show it is unique (as far as we know) in being able to scale beyond the bounds of main memory.
\end{abstract}

\section{Introduction}

FDR (Failures Divergence Refinement) is the most widespread refinement checker for the process algebra CSP 123. FDR takes a list of CSP processes, written in machine-readable CSP (henceforth $\mathrm{CSP}_{M}$ ) which is a lazy functional language, and is able to check if the processes refine each other according to the CSP denotational models (e.g. the traces, failures and failures-divergences models). It is also able to check for more properties, including deadlock-freedom, livelock-freedom and determinism, by constructing equivalent refinement checks.

FDR2 was released in 1996, and has been widely used both within industry and in academia for verifying systems [4,56]. It is also used as a verification backend for several other tools including: Casper [7] which verifies security protocols; SVA [8] which can verify simple shared-variable programs; in addition to several industrial tools (e.g. ModelWorks and ASD).

FDR3 has been under development for the last few years as a complete rewrite of FDR2. It represents a major advance over FDR2, not only in the size of system that can be checked (we have verified systems with over ten billion states in a few hours), but also in terms of its ease of use. FDR3 has also been designed and engineered to be a stable platform for future development of CSP modelchecking tools, in addition to tools for CSP-like languages [2]. In this paper we give an outline of FDR3, highlighting a selection of the advances made.

E. Ábrahám and K. Havelund (Eds.): TACAS 2014, LNCS 8413, pp. 187-201, 2014.

(C) Springer-Verlag Berlin Heidelberg 2014 
In Section 4 we describe the new multi-core refinement-checking algorithm that achieves a near linear increase in performance as the number of cores increases. Section 6 gives some experimental results that compare the performance of the new algorithm to FDR2, Spin [9], DiVinE [10, and LTSmin [11.

In Section 5 we detail the new compilation algorithm, which constructs FDR's internal representation of CSP processes (i.e. labelled-transition systems) from $\mathrm{CSP}_{M}$ processes. This algorithm is an entirely new development and is able to compile many CSP processes into more efficient labelled-transition systems. It is also related to the operational semantics of CSP, unlike the FDR2 algorithm which was based on heuristics.

In addition to the advances that we present in this paper, FDR3 incorporates a number of other new features. Most notably, the graphical user interface has been entirely rethought, and includes: a new $\mathrm{CSP}_{M}$ type checker; a built-in version of ProBE, the CSP process animator; and a new debugger that emphasises interactions between processes. See the FDR3 manual [12] for further details.

Before describing the new advances, in Section 2 we briefly review CSP. In Section 3 we then outline the high-level design and structure of FDR3.

\section{CSP}

CSP [1/2]3] is a process algebra in which programs or processes that communicate events from a set $\Sigma$ with an environment may be described. We sometimes structure events by sending them along a channel. For example, c.3 denotes the value 3 being sent along the channel $c$. Further, given a channel $c$ the set $\{c \mid\} \subseteq \Sigma$ contains those events of the form c.x.

The simplest CSP process is the process STOP that can perform no events. The process $a \rightarrow P$ offers the environment the event $a \in \Sigma$ and then behaves like $P$. The process $P \square Q$ offers the environment the choice of the events offered by $P$ and by $Q$ and is not resolved by the internal action $\tau$. $P \sqcap Q$ non-deterministically chooses which of $P$ or $Q$ to behave like. $P \triangleright Q$ initially behaves like $P$, but can timeout (via $\tau$ ) and then behaves as $Q$.

$P{ }_{A} \|_{B} Q$ allows $P$ and $Q$ to perform only events from $A$ and $B$ respectively and forces $P$ and $Q$ to synchronise on events in $A \cap B$. $P \| Q$ allows $P$ and $Q$ to A

run in parallel, forcing synchronisation on events in $A$ and arbitrary interleaving of events not in $A$. The interleaving of two processes, denoted $P \| \mid Q$, runs $P$ and $Q$ in parallel but enforces no synchronisation. $P \backslash A$ behaves as $P$ but hides any events from $A$ by transforming them into the internal event $\tau$. This event does not synchronise with the environment and thus can always occur. $P[R]$, behaves as $P$ but renames the events according to the relation $R$. Hence, if $P$ can perform $a$, then $P[[R]$ can perform each $b$ such that $(a, b) \in R$, where the choice (if more than one such $b$ ) is left to the environment (like $\square$ ). $P \triangle Q$ initially behaves like $P$ but allows $Q$ to interrupt at any point and perform a visible event, at which point $P$ is discarded and the process behaves like $Q . P \Theta_{A} Q$ initially behaves like $P$, but if $P$ ever performs an event from $A, P$ is discarded and $P \Theta_{A} Q$ behaves like $Q$. Skip is the process that immediately terminates. 
The sequential composition of $P$ and $Q$, denoted $P ; Q$, runs $P$ until it terminates at which point $Q$ is run. Termination is indicated using a $\checkmark$ : Skip is defined as $\checkmark \rightarrow S T O P$ and, if the left argument of $P$; $Q$ performs a $\checkmark, P ; Q$ performs a $\tau$ to the state $Q$ (i.e. $P$ is discarded and $Q$ is started).

Recursive processes can be defined either equationally or using the notation $\mu X \cdot P$. In the latter, every occurrence of $X$ within $P$ represents a recursive call.

An argument $P$ of a CSP operator $O p$ is on iff it can perform an event. $P$ is off iff no such rule exists. For example, the left argument of the exception operator is on, whilst the right argument is off.

The simplest approach to giving meaning to a CSP expression is by defining an operational semantics. The operational semantics of a CSP process naturally creates a labelled transition system (LTS) where the edges are labelled by events from $\Sigma \cup\{\tau\}$ and the nodes are process states. Formally, an LTS is a 3-tuple consisting of a set of nodes, an initial node, and a relation $\stackrel{a}{\longrightarrow}$ on the nodes: i.e. it is a directed graph where each edge is labelled by an event. The usual way of defining the operational semantics of CSP processes is by presenting Structured Operational Semantics (SOS) style rules in order to define $\stackrel{a}{\longrightarrow}$. For instance, the operational semantics of the exception operator are defined by:

$$
\frac{P \stackrel{a}{\longrightarrow} P^{\prime}}{P \Theta_{A} Q \stackrel{a}{\longrightarrow} Q} a \in A \frac{P \stackrel{b}{\longrightarrow} P^{\prime}}{P \Theta_{A} Q \stackrel{b}{\longrightarrow} P^{\prime} \Theta_{A} Q} b \notin A \frac{P \stackrel{\tau}{\longrightarrow} P^{\prime}}{P \Theta_{A} Q \stackrel{\tau}{\longrightarrow} P^{\prime} \Theta_{A} Q}
$$

The interesting rule is the first, which specifies that if $P$ performs an event $a \in A$, then $P \Theta_{A} Q$ can perform the event $a$ and behave like $Q$.

The SOS style of operational semantics is far more expressive than is required to give an operational semantics to CSP, and indeed can define operators which, for a variety of reasons, make no sense in CSP models. As pointed out in [3], it is possible to re-formulate CSP's semantics in the highly restricted combinator style of operational semantics, which largely concentrates on the relationships between events of argument processes and those of the constructed system. This style says, inter alia, that only on arguments can influence events, that any $\tau$ action of an on argument must be allowed to proceed freely, and that an argument process has changed state in the result state if and only if it has participated in the action. Cloning of on arguments is not permitted. Any language with a combinator operational semantics can be translated to CSP with a high degree of faithfulness [3] and is compositional over every CSP model. FDR3 is designed so that it can readily be extended to such CSP-like languages.

CSP also has a number of denotational models, such as the traces, failures and failures-divergences models. In these models, each process is represented by a set of behaviours: the traces model represents a process by the set of sequences of events it can perform; the failures model represents a process by the set of events it can refuse after each trace; the failures-divergences model augments the failures model with information about when a process can perform an unbounded number of $\tau$ events. Two processes are equal in a denotational model iff they have the same set of behaviours. If every behaviour of $I m p l$ is a behaviour of Spec in the denotational model $X$, then Spec is refined by Impl, denoted Spec $\sqsubseteq_{X}$ Impl. 


\section{The Overall Structure of FDR3}

As FDR3 is a refinement checker (deadlock freedom, determinism, etc. are converted into refinement checks), we consider how FDR3 decides if $P \sqsubseteq Q$.

Since $P$ and $Q$ will actually be $\mathrm{CSP}_{M}$ expressions, FDR3 needs to evaluate them to produce a tree of CSP operator applications. For example, if $P$ was the $\mathrm{CSP}_{M}$ expression if true then $c$ ?x $\rightarrow$ STOP else STOP, this would evaluate to c.0 -> STOP [ ] c.1 $\rightarrow$ STOP. Notice that the functional language has been removed: all that remains is a tree of trivial operator applications, as follows.

Definition 1. A syntactic process $P$ is generated according to the grammar: $P::=\operatorname{Operator}\left(P_{1}, \ldots, P_{M}\right) \mid N$ where the $P_{i}$ are also syntactic processes, Operator is any CSP operator (e.g. external choice, prefix etc) and $N$ is a process name. A syntactic process environment $\Gamma$ is a function from process name to syntactic process such that $\Gamma(N)$ is never a process name.

The evaluator converts $\operatorname{CSP}_{M}$ expressions to syntactic processes. Since $\operatorname{CSP}_{M}$ is a lazy functional language, the complexity of evaluating $\mathrm{CSP}_{M}$ depends on how the $\mathrm{CSP}_{M}$ code has been written. This is written in Haskell and is available as part of the open-source Haskell library libcspm [13], which implements a parser, type-checker and evaluator for $\mathrm{CSP}_{M}$.

Given a syntactic process, FDR3 then converts this to an LTS which is used to represent CSP processes during refinement checks. In order to support various features (most importantly, the compressions such as normalisation), FDR internally represents processes as generalised labelled transition systems (GLTSs), rather than LTSs. These differ from LTSs in that the individual states can be labelled with properties according to the semantic model in use. For example, if the failures model is being used, a GLTS would allow states to be labelled with refusals. The compiler is responsible for converting syntactic processes into GLTSs. The primary challenge for the compiler is to decide which of FDR3's internal representations of GLTSs (which have various trade-offs) should be used to represent each syntactic process. This algorithm is detailed in Section 5 .

After FDR3 has constructed GLTSs for the specification and implementation processes, FDR3 checks for refinement. Firstly, as with FDR2, the specification GLTS is normalised [3] to yield a deterministic GLTS with no $\tau$ 's. Normalising large specifications is expensive, however, generally specifications are relatively small. FDR3 then checks if the implementation GLTS refines the normalised specification GLTS according to the algorithm presented in Section 4.

Like FDR2, FDR3 supports a variety of compressions which can be used to cut the state space of a system. FDR3 essentially supports the compressions of [3], in some cases with significantly improved algorithms, which we will report on separately. It also supports the chase operator of FDR2 which forces $\tau$ actions and is a useful pruner of state spaces where it is semantically valid.

Like recent versions of FDR2, FDR3 supports the Timed CSP language 14 15. It uses the strategy outlined in [16]3] of translating the continuous Timed CSP language to a variant of untimed CSP with prioritisation and relying on 


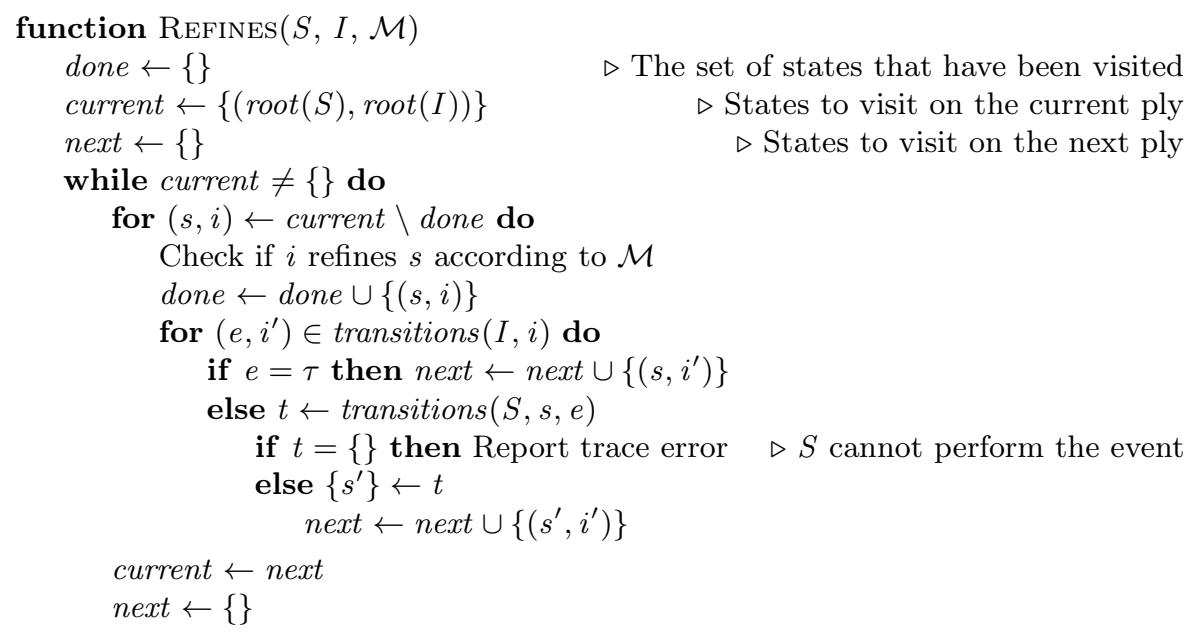

Fig. 1. The single-threaded refinement-checking algorithm where: $S$ is the normalised specification GLTS; $I$ is the implementation GLTS; $\mathcal{M}$ is the denotational model to perform the check in; $\operatorname{root}(X)$ returns the root of the GLTS $X$; $\operatorname{transitions}(X, s)$ returns the set of all $\left(e, s^{\prime}\right)$ such that there is a transition from $s$ to $s^{\prime}$ in the GLTS $X$ labelled by the event $e$; transitions $(X, s, e)$ returns only successors under event $e$

theorems of digitisation [17]. In order to support this, FDR3 also supports the prioritise operator 318, which has other interesting applications as shown there.

\section{Parallel Refinement Checking}

We now describe the new multi-core refinement-checking algorithm that FDR3 uses to decide if a normalised GLTS $P$ (recall that normalisation produces a GLTS with no $\tau$ 's and such that for each state and each event, there is a unique successor state) is refined by another GLTS $Q$. We begin by outlining the refinement checking algorithm of [2] and describing the FDR2 implementation [19]. We then define the parallel refinement-checking algorithm, before contrasting our approach with the approaches taken by others to parallelise similar problems.

In this paper we concentrate on parallelising refinement checking on sharedmemory systems. We also concentrate on refinement checking in models that do not consider divergence: we will report separately on parallelising this.

The Single-Threaded Algorithm Refinement checking proceeds by performing a search over the implementation, checking that every reachable state is compatible with every state of the specification after the same trace. A breadth-first search is performed since this produces a minimal counterexample when the check fails. The single threaded algorithm [2]19] is given in Figure 1$]$

The interesting aspect of an implementation of the above algorithm is how it stores the sets of states (i.e. current, next and done). FDR2 uses B-Trees for 


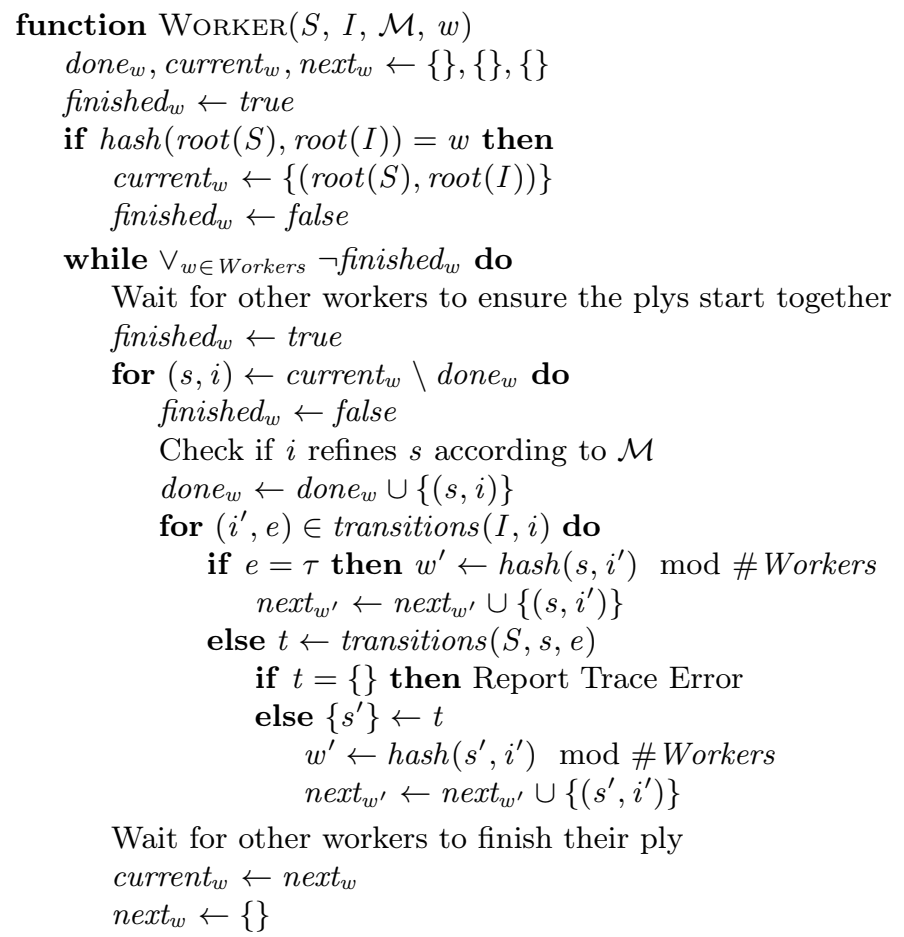

Fig. 2. Each worker in a parallel refinement check executes the above function. The set of all workers is given by Workers. $\operatorname{Hash}(s, i)$ is an efficient hash function on the state pair $(s, i)$. All other functions are as per Figure 1

all of the above sets 19, primarily because this allowed checks to efficiently use disk-based storage when RAM was exhausted (in contrast to, e.g. hash tables, where performance often decays to the point of being unusable once RAM has been exhausted). This brings the additional benefit that inserts into done (from current) can be performed in sorted order. Since B-Trees perform almost optimally under such workloads, this makes insertions into the done tree highly efficient. To improve efficiency, inserts into the next tree are buffered, with the buffer being sorted before insertion. The storage that the B-Tree uses is also compressed, typically resulting in memory requirements being halved.

Parallelisation Parallelising FDR3's refinement checking essentially reduces to parallelising the breadth-first search of Figure 1. Our algorithm partitions the state space based on a hash function on the node pairs. Each worker is assigned a partition and has local current, next and done sets. When a worker visits a transition, it computes the worker who is responsible for the destination by hashing the new state pair. This algorithm is presented in Figure 2.

Whilst the abstract algorithm is straightforward, the implementation has to be carefully designed in order to obtain good performance. As before, our primary 
consideration is minimising memory usage. In fact, this becomes even more critical in the parallel setting since memory will be consumed at a far greater rate: with 16 cores, FDR3 can visit up to 7 billion states per hour consuming 70GB of storage. Thus, we need to allow checks to exceed the size of the available RAM. Given the above, B-Trees are a natural choice for storing the sets.

All access to the done and current B-Trees is restricted to the worker who owns those B-Trees, meaning that there are no threading issues to consider. The next B-Trees are more problematic: workers can generate node pairs for other workers. Thus, we need to provide some way of accessing the next B-Trees of other workers in a thread-safe manner. Given the volume of data that needs to be put into next (which can be an order of magnitude greater than the volume put into done), locking the tree is undesirable. One option would be to use finegrained locking on the B-Tree, however this is difficult to implement efficiently.

Instead of using complex locks, we have generalised the buffering that is used to insert into next under the single-threaded algorithm. Each worker $w$ has a set of buffers, one for each other worker, and a list of buffers it has received from other workers that require insertion into this worker's next. When a buffer of worker $w$ for worker $w^{\prime} \neq w$ fills up, it immediately passes it to the target worker. Workers periodically check the stack of pending buffers to be flushed, and when a certain size is exceeded, they perform a bulk insert into next by performing a $n$-way merge of all of the pending buffers to produce a single sorted buffer.

One potential issue this algorithm could suffer from is uneven distribution amongst the workers. We have not observed this problem: the workers have terminated at roughly the same time. If necessary this could be addressed by increasing the number of partitions, with workers picking a partition to work on.

We give experimental results that show the algorithm is able to achieve a near linear speed up in Section 6 .

Related Work There have been many algorithms proposed for parallelising BFS, e.g. [20 21 22 23]. In general, these solutions do not attempt to optimise memory usage of performance once RAM has been exhausted to the same degree.

The authors of [20] parallelised the FDR2 refinement checker for cluster systems that used MPI. The algorithm they used was similar to our algorithm in that nodes were partitioned amongst the workers and that B-Trees were used for storage. The main difference comes from the communication of next: in their approach this was deferred until the end of each round where a bulk exchange was done, whereas in our model we use a complex buffer system.

The authors of [21] propose a solution that is optimised for performing a BFS on sparse graphs. This uses a novel tree structure to efficiently (in terms of time) store the bag of nodes that are to be visited on the next ply. This was not suitable for FDR since it does not provide a general solution for eliminating duplicates in next, which would cause FDR3 to use vastly more memory.

The author of [23] enhances the Spin Model Checker 9] to support parallel BFS. In this solution, which is based on [24], done is a lock-free hash-table and is shared between all of the workers, whilst new states are randomly assigned to a number of subsets which are lock-free linked lists. This approach is not suitable 
for FDR since hash-tables are known not to perform well once RAM has been exhausted (due to their essentially random access pattern). Storing next in a series of linked-lists is suitable for Spin since it can efficiently check if a node is in done using the lock-free hash-table. This is not the case for FDR, since there is no way of efficiently checking if a node is in the done B-Tree of a worker.

\section{Compiler}

As outlined in Section 3, the compiler is responsible for converting syntactic processes into GLTSs. This is a difficult problem due to the generality of CSP since operators can be combined in almost arbitrary ways. In order to allow the processes to be represented efficiently, FDR3 has a number of different GLTS types as described in Section [5.1, and a number of different way of constructing each GLTS, as described in Section 5.2 In Section 5.3 we detail the new algorithm that the compiler uses to decide which of FDR3's representations of GLTSs to use. This is of critical importance: if FDR3 were to choose the wrong representation this could cause the time to check a property and the memory requirements to greatly increase.

\section{$5.1 \quad$ GLTSs}

FDR3 has two main representations of GLTSs: Explicit and Super-Combinator machines. Explicit machines require memory proportional to the number of states and transitions during a refinement check. In contrast, Super-Combinator machines only require storage proportional to the number of states, since the transitions can be computed on-the-fly. Equally, it takes longer to calculate the transitions of a SuperCombinator machine than the corresponding Explicit machine.

An Explicit GLTS is simply a standard graph data structure. Nodes in an Explicit GLTS are process states whilst the transitions are stored in a sorted list. A Super-Combinator machine represents the LTS by a series of component LTSs along with a list of rules to combine the transitions of the components. Nodes for a Super-Combinator machine are tuples, with one entry for each component machine. For example, a Super-Combinator for $P\|\| Q$ consists of the components $\langle P, Q\rangle$ and the rules:

$$
\{(\langle 1 \mapsto a\rangle, a) \mid a \in \alpha P \cup\{\tau\}\} \cup\{(\langle 2 \mapsto a\rangle, a) \mid a \in \alpha Q \cup\{\tau\}\}
$$

where $\alpha X$ is the alphabet of the process $X$ (i.e. the set of events it can perform). These rules describe how to combine the actions of $P$ and $Q$ into actions of the whole machine. A single rule is of the form $(f, e)$ where $f$ is a partial function from the index of a component machine (e.g. in the above example, 1 represents $P)$ to the event that component must perform. $e$ is the event the overall machine performs if all components perform their required events.

Rules can also be split into formats, which are sets of rules. For example, a Super-Combinator for $P$; $Q$ would start in format 1, which has the rules:

$$
\{(\langle 1 \mapsto a\rangle, a, 1) \mid a \in \alpha P \cup\{\tau\}, a \neq \checkmark\} \cup\{(\langle 1 \mapsto \checkmark\rangle, \tau, 2) \mid a \in \alpha Q \cup\{\tau\}\} .
$$


The second format has the rules: $\{(\langle 2 \mapsto a\rangle, a, 2) \mid a \in \alpha Q \cup\{\tau\}\}$. Thus, the first format allows $P$ to perform visible events and stay in format 1 (as indicated by the third element of the tuple), but if $P$ performs a $\checkmark$ and terminates, the second format is started which allows $Q$ to perform visible events.

Rules can also specify that component machines should be restarted. For example, to represent $P=X ; P$ as a Super-Combinator, there needs to be a way of restarting the process $X$ after a $\checkmark$. Thus, we add to the rules a list of components whose states should be discarded and replaced by their root states:

$$
\{(\{1 \mapsto a\}, a, 1,\langle\rangle) \mid a \in \alpha X \cup\{\tau\}, a \neq \checkmark)\} \cup\{(\{1 \mapsto \checkmark\}, \tau, 1,\langle 1\rangle)\} .
$$

The first rule set allows $X$ to perform non- $\checkmark$ events as usual. However, if $X$ ever performs a $\checkmark$ this is converted into a $\tau$ and component 1 (i.e. $X$ ) is restarted.

FDR also recursively combines the rules for Super-Combinator machines. For example, $(P \| Q) \| R$ is not represented as two different Super-Combinator machines, but instead the rules for $P\|\| Q$ and $\cdot \| R$ are combined. This process is known as supercompilation. As you might expect from the name, supercombinators are closely related to combinator operational semantics: the "super" essentially co-incides with the joining together using supercompilation.

\subsection{Strategies}

There are several different strategies that FDR3 can use to construct Explicit or Super-Combinator machines from syntactic processes. These strategies differ in the type of processes that they can support (e.g. some cannot support recursive processes), the time they take to execute and the type of the resulting GLTS.

The low-level is the simplest strategy and supports any process. An Explicit LTS is constructed simply by directly applying CSP's operational semantics.

The high-level compiles a process to a Super-Combinator. This is not able to compile recursive processes, such as $P \widehat{=} a \rightarrow P$. The supercombinator rules are directly constructed using the operational semantics of CSP.

The mixed-level is a hybrid of the low and high-level strategies where, intuitively, non-recursive parts of processes are compiled as per the high-level strategy whilst recursive parts are compiled as per the low-level strategy. For example, consider $P \widehat{=} a \rightarrow P \square b \rightarrow(X \| Y)$ : compiling $X \| \mid Y$ at the high-level is preferable since it does not require the cartesian product of $X$ and $Y$ to be formed. If $P$ is compiled at the mixed-level, $X\|\| Y$ is compiled at the high-level, and $a \rightarrow P \square b \rightarrow \cdot$ is compiled into an Explicit machine. These are wrapped in a Super-Combinator machine that starts $X \| \mid Y$ when the Explicit machine performs the $b$. The supercombinator has two formats, the first with the rules: $\{(\{1 \mapsto a\}, a, 1),(\{1 \mapsto b\}, b, 2)\}$ and the second with: $\{(\{\mathscr{2} \mapsto a\}, a, 2) \mid a \in \alpha(X|| \mid Y) \cup\{\tau\}\}$. Thus, when the first process performs $b$, the Super-Combinator moves to the second format in which $X\|\| Y$ is run. The next section formalises the set of process that can be compiled in this way.

The recursive high-level strategy is new in FDR3. This compiles to a SuperCombinator machine and allows some recursive processes (which we formalise 
in the next section) to be compiled. This is used to compile processes such as $P \widehat{=}(X \| Y) ; P$ which are recursive, but are desirable to compile to SuperCombinator machines for efficiency reasons (as above, constructing $X\|\| Y$ is expensive). In this particular case, $X\|\| Y$ is compiled to a Super-Combinator machine, and then a recursive supercombinator is constructed with the rules:

$$
\{(\{1 \mapsto a\}, a, 1,\langle\rangle) \mid a \in \alpha(X \mid \| Y) \cup\{\tau\}, a \neq \checkmark)\} \cup\{(\{1 \mapsto \checkmark\}, \tau, 1,\langle 1\rangle)\} .
$$

Recall that the last component in the above rules indicates that component 1 should be reset. Thus, the above rules indicate that $X \||| Y$ can perform non- $\checkmark$ events normally, but a $\checkmark$ will cause $X \| \mid Y$ to be reset to its initial state.

The majority of processes can be compiled at the recursive high-level, with the exception of those that recurse through an on argument of an operator (e.g. $P=a \rightarrow P \square b \rightarrow P)$. For example, consider the process $P=X ;(P \square \ldots)$ : since $\square$ is not discarded by a $\tau$, it follows that this recursion is safe only when $X$ always performs a visible event before a $\checkmark$ (otherwise there would be an infinite series of $\square$ 's applied). This cannot be determined statically (i.e. without accessing the transitions of $X$ ), and thus it is not possible to determine if the process can be compiled at the recursive high-level. Thankfully, such processes are sufficiently rare in the context where recursive high-level is of use.

\subsection{Picking a Strategy}

We now describe the new algorithm that FDR3 uses to decide how to compile a syntactic process. The input to the compilation algorithm is a syntactic process environment (Definition 1) and the output is a list of strategies that specify how each syntactic processes should be compiled. The algorithm guarantees to produce a strategy such that executing the strategy yields a valid GLTS that corresponds to the input process. The algorithm also uses heuristics to attempt to reduce the time and memory usage during the subsequent refinement check.

All operators have a preferred level of compilation, either low (indicating Explicit is preferred) or high (indicating Super-Combinator is preferred). For example, prefix prefers the low whilst interleave prefers high. In general, FDR3 aims to compile an operator at its preferred level. If this is high, this may require using the mixed and recursive high-level strategies on surrounding processes (a preference for high is more important). When this is not possible (because, e.g., the processes do not permit the mixed level), the low-level strategy is used.

The first step is to calculate the strongly connected components (SCCs) of recursive processes. This is done by performing a DFS on the recursion graph that is naturally formed from the syntactic process environment. Then, we compute which SCCs can be compiled at the recursive high-level, and which SCCs would prefer to be compiled at the recursive high-level (by incorporating preferences, e.g. prefix prefers to recurse at low, but; prefers high). The graph is also used to check for invalid processes, such as $P=P \square P$ : formally, for each process name $P$ we check that on each path back to $P$, at least one off argument is traversed.

Using the recursion graph, FDR3 computes which strategy to use to compile a syntactic process $P$. This cannot be done in ignorance of the context of $P$, 


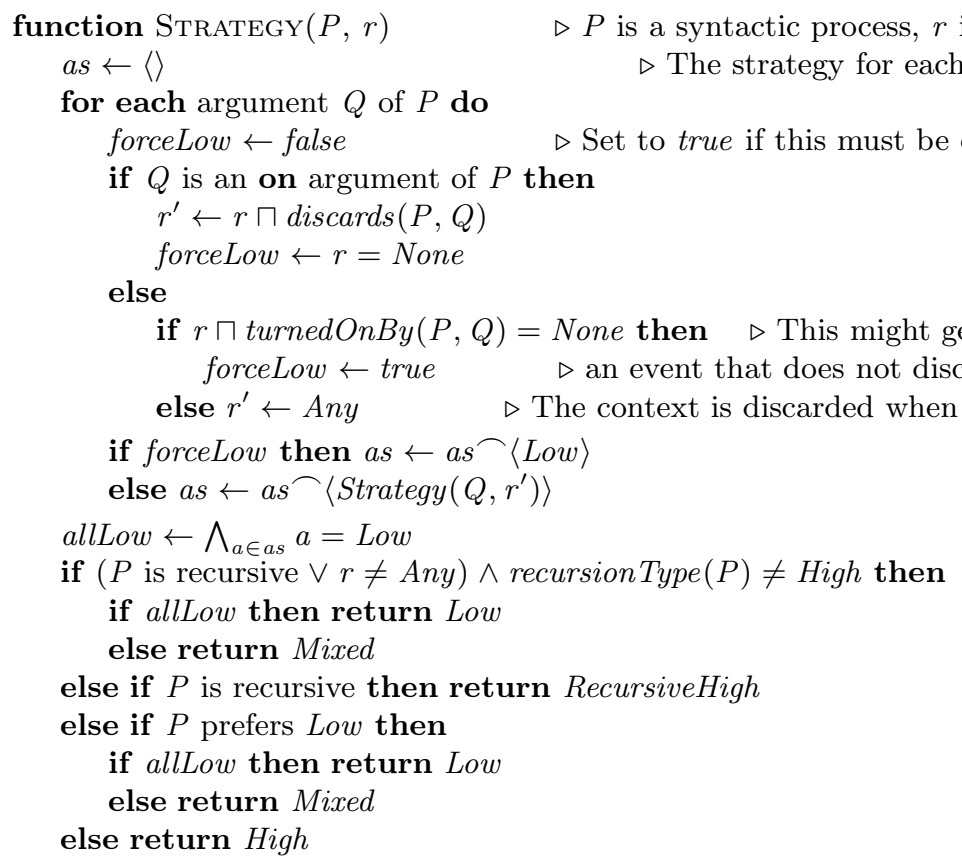

Fig. 3. The algorithm FDR3 uses to decide how to compile syntactic processes

since this may dictate how a process is compiled. For example, $P=a \rightarrow P \square Q$ requires $Q$ to be compiled at the low-level, since $P$ is a low-level recursion and $Q$ appears as an on argument of an operator that is on the recursion path. Thus, when compiling a syntactic process, we need to be aware of the surrounding context $C[\cdot]$, (e.g. $\left.C_{1}[X] \widehat{=} X \| S T O P\right)$. When deciding on the strategy for $P$, the relevant fact about the context is what events $P$ can perform to cause the context to be discarded. For example, nothing can discard the context $C_{1}$, whilst any visible event discards the context $C_{2}[X] \widehat{=} X \square S T O P$. As we are interested in statically analysing processes, we approximate these sets as follows.

Definition 2. An event type is either None, Invisible, Visible or Any. The relation $<$ is defined as None $<$ Invisible, None $<$ Visible, Invisible $<$ Any, Visible $<$ Any. Note $<$ is a partial order on event types. The meet of $e_{1}$ and $e_{2}$ is denoted by $e_{1} \sqcap e_{2}$.

Definition 3. Let $Q$ be an argument of a syntactic process $P$. If $Q$ is on, then $\operatorname{discards}(P, Q)$ returns the event type that $Q$ performs to cause $P$ to be discarded and $Q$ to be left running (e.g. discards $(X \square Y, X)=$ Visible, whilst $\operatorname{discards}(X \| Y, X)=$ None $)$. If $Q$ is off, then turnedOnBy $(P, Q)$ returns the event type that $P$ performs in order to turn on $Q$. For example, turnedOnBy $(X$; $Y, Y)=$ Invisible whilst turnedOnBy $(X \Theta . Y, Y)=$ Visible.

Thus it is possible to use discards along with the meet on event types to compute when a context will be discarded. 
Figure3defines a function Strategy $(P, r)$ that returns the strategy that should be used to compile the syntactic process $P$ in a context that is discarded by events of event type $r$. Informally, given a process $P$ and an event type $r$ this firstly recursively visits each of its arguments, passing down an appropriate event restriction (which is computed using discards for on arguments and turnedOnBy for off arguments). It may also force some arguments to be low-level if the restriction becomes None. Then, a compilation strategy for $P$ is computed by considering the preferences of the operator, whether the operator is recursive and the deduced strategies for the arguments. The overriding observation behind this choice is that compilation at high is only allowed when the process is nonrecursive, and when there is no surrounding context (i.e. $r=$ Anything).

\subsection{Related Work}

FDR2 has support for Explicit and Super-Combinator GLTSs, along with a GLTS definition for each CSP operator (e.g. external choice etc). We believe that the FDR3 representation is superior, since it requires fewer GLTS types to be maintained and because it makes the GLTSs independent of CSP, making other process algebras easier to support. As mentioned in Section 5.2. FDR2 did not make use of the recursive high-level, and was unable to automatically compile processes such as $P=(X \| Y) ; P$ at the high-level. We have found that the recursive high-level has dramatically decreased compilation time on many examples.

The biggest difference is in the algorithm that each uses to compile syntactic processes. FDR2 essentially used a series of heuristics to accomplish this and would always start trying to compile the process at its preferred level, backtracking where necessary. This produced undesirable behaviour on certain processes. We believe that since the new algorithm is based on the operational semantics of CSP, it is simpler and can be easily applied to other CSP-like process algebras.

\section{Experiments}

We compare the performance of a pre-release version of FDR 3.1.0 to FDR 2.94, Spin 6.25, DiVinE 3.1 beta 1, and LTSmin 2.0, on a complete traversal of a graph. The experiments were performed on a Linux server with two 8 core $2 \mathrm{GHz}$ Xeons with hyperthreading (i.e. 32 virtual cores), 128GB RAM, and five 100GB SSDs. All input files are available from the first author's webpage. - denotes a check that took over 6 hours, ${ }^{*}$ denotes a check that was not attempted, and $\dagger$ denotes a check that could not be completed due to insufficient memory. Times refer to the total time required to run each program whilst memory figures refer to the maximum Resident Set Size plus any on-disk storage used.

Figure 4a compares the performance of FDR2 and FDR3. FDR3 with 1 worker substantially outperforms FDR2. This is because FDR3's B-Tree has been heavily optimised and because FDR3 makes fewer allocations during refinement checks. FDR3 with 1 worker also uses less memory than FDR2: this is due to a new compaction algorithm used to compress B-Tree blocks that only 


\begin{tabular}{|c|c|c||c|c|c|c|}
\hline \multirow{2}{*}{ Input } & \multirow{2}{*}{$\begin{array}{c}\text { States } \\
\end{array}$} & Transitions & \multicolumn{2}{|c|}{ Time (s) \& Storage (GB) } & FDR3-32 \\
\cline { 4 - 7 } & $\left(10^{6}\right)$ & $\left(10^{6}\right)$ & FDR2 & FDR3-1 & FDR3-32 & Speedup \\
\hline bully.7 & 129 & 1354 & $2205(4.8)$ & $1023(2.2)$ & $85(5.5)$ & 12.0 \\
cuberoll.0 & 7524 & 20065 & - & - & $3546(74.5)$ & - \\
ddb.0 & 65 & 377 & $722(1.4)$ & $405(0.5)$ & $31(2.36)$ & 13.1 \\
knightex.5.5 & 67 & 259 & $550(1.4)$ & $282(0.6)$ & $23(2.4)$ & 12.3 \\
knightex.3.11 & 19835 & 67321 & $*$ & $*$ & $26235(298.5)$ & - \\
phils.10 & 60 & 533 & $789(1.3)$ & $431(0.5)$ & $32(2.0)$ & 13.5 \\
solitare.0 & 187 & 1487 & $2059(4.4)$ & $1249(1.6)$ & $84(3.8)$ & 14.9 \\
solitare.1 & 1564 & 13971 & $19318(35.1)$ & $11357(11.7)$ & $944(17.5)$ & 12.0 \\
solitaire.2 & 11622 & 113767 & $*$ & $*$ & $9422(113.3)$ & - \\
tnonblock.7 & 322 & 635 & $2773(6.7)$ & $937(2.6)$ & $109(6.8)$ & 8.6 \\
\hline
\end{tabular}

(a) Times comparing FDR2, FDR3 with 1 worker, and FDR3 with 32 workers.

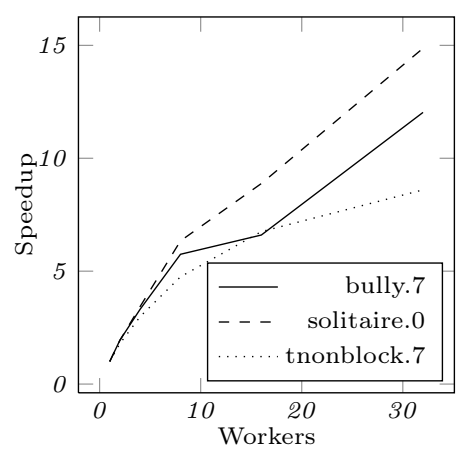

(b) FDR3's scaling performance.

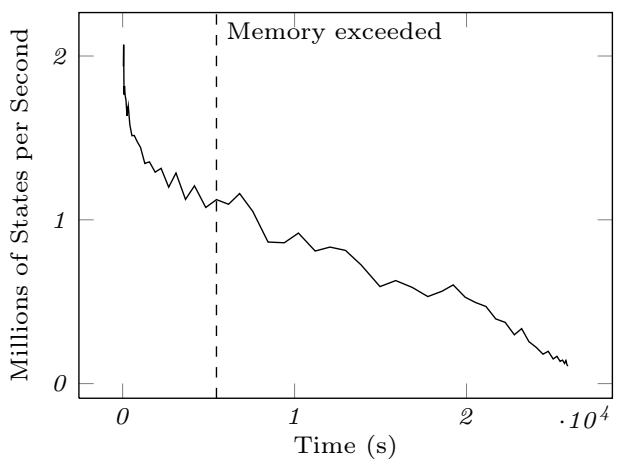

(c) Disk storage performance on knightex.3.11.

Fig. 4. Experimental results demonstrating FDR3's performance

stores the difference between keys. The extra memory used for the parallel version is for extra buffers and the fact that the B-Tree blocks do not compress as well.

The speed-ups that Figures $4 \mathrm{a}$ and $4 \mathrm{~b}$ exhibit between 1 and 32 workers vary according to the problem. solitaire is sped up by a factor of 15 which is almost optimal given the 16 cores. Conversely, tnonblock. 7 is only sped up by a factor of 9 because it has many small plys, meaning that the time spent waiting for other workers at the end of a ply is larger.

Figure 4c shows how the speed that FDR3 visits states at changes during the course of verifying knightex.3.11, which required 300GB of storage (FDR3 used $110 \mathrm{~GB}$ of memory as a cache and $190 \mathrm{~GB}$ of on-disk storage). During a refinement check, the rate at which states are explored will decrease because the B-Trees increase in size. Observe that there is no change in the decrease of the state visiting rate after memory is exceeded. This demonstrates that B-Trees are effectively able to scale to use large amounts of on-disk storage.

Figure 5 compares the performance of FDR3, Spin, DiVinE and LTSmin. For in-memory checks Spin, DiVinE and LTSmin complete the checks up to three 


\begin{tabular}{|c|c|c|c|c|}
\hline \multirow{2}{*}{ Input } & \multicolumn{4}{|c|}{ Time (s) \& Storage (GB) } \\
\cline { 2 - 5 } & Spin-32 & FDR3-32 & DiVinE-32 & LTSmin-32 \\
\hline knightex.5.5 & $12(5.8)$ & $23(2.4)$ & $13(4.6)$ & $28(33.1)$ \\
knightex.3.10 & $396(115.0)$ & $943(22.7)$ & $\dagger$ & $395(35.5)$ \\
knightex.3.11 & $\dagger$ & $26235(298.5)$ & $\dagger$ & $\dagger$ \\
solitaire.0 & $89(15.5)$ & $85(3.9)$ & $85(14.3)$ & $73(36.4)$ \\
\hline
\end{tabular}

Fig. 5. A comparison between FDR3, Spin, DiVinE and LTSmin. knightex.3.10 has $2035 \times 10^{6}$ states and $6786 \times 10^{6}$ transitions.

times faster than FDR3 but use up to four times more memory. We believe that FDR3 is slower because supercombinators are expensive to execute in comparison to the LTS representations that other tools use, and because B-Trees are slower to insert into than hashtables. FDR3 was the only tool that was able to complete knightex.3.11 which requires use of on-disk storage; Spin, DiVinE and LTSmin were initially fast, but dramatically slowed once main memory was exhausted.

\section{Conclusions}

In this paper we have presented FDR3, a new refinement checker for CSP. We have described the new compiler that is more efficient, more clearly defined and produces better representations than the FDR2 compiler. Further, we have detailed the new parallel refinement-checking algorithm that is able to achieve a near-linear speed-up as the number of cores increases whilst ensuring efficient memory usage. Further, we have demonstrated that FDR3 is able to scale to enormous checks that far exceed the bounds of memory, unlike related tools.

This paper concentrates on parallelising refinement checks on shared-memory systems. It would be interesting to extend this to support clusters instead: this would allow even larger checks to be run. It would also be useful to consider how to best parallelise checks in the failures-divergence model. This is a difficult problem, in general, since this uses a depth-first search to find cycles.

FDR3 is available for 64-bit Linux and Mac OS $\mathrm{X}$ from https://www. cs.ox.ac.uk/projects/fdr/. FDR3 is free for personal use or academic research, whilst commercial use requires a licence.

Acknowledgements. This work has benefitted from many useful conversations with Michael Goldsmith, Colin O'Halloran, Gavin Lowe, and Nick Moffat. We would also like to thank the anonymous reviewers for their useful comments.

\section{References}

1. Hoare, C.A.R.: Communicating Sequential Processes. Prentice-Hall, Inc., Upper Saddle River (1985)

2. Roscoe, A.W.: The Theory and Practice of Concurrency. Prentice Hall (1997) 
3. Roscoe, A.W.: Understanding Concurrent Systems. Springer (2010)

4. Lawrence, J.: Practical Application of CSP and FDR to Software Design. In: Abdallah, A.E., Jones, C.B., Sanders, J.W. (eds.) CSP25. LNCS, vol. 3525, pp. 151-174. Springer, Heidelberg (2005)

5. Mota, A., Sampaio, A.: Model-checking CSP-Z: strategy, tool support and industrial application. Science of Computer Programming 40(1) (2001)

6. Fischer, C., Wehrheim, H.: Model-Checking CSP-OZ Specifications with FDR. In: IFM 1999. Springer (1999)

7. Lowe, G.: Casper: A Compiler for the Analysis of Security Protocols. Journal of Computer Security 6(1-2) (1998)

8. Roscoe, A.W., Hopkins, D.: SVA, a Tool for Analysing Shared-Variable Programs. In: Proceedings of AVoCS 2007 (2007)

9. Holzmann, G.: Spin Model Checker: The Primer and Reference Manual. AddisonWesley Professional (2003)

10. Barnat, J., Brim, L., Havel, V., Havlíček, J., Kriho, J., Lenčo, M., Ročkai, P., Štill, V., Weiser, J.: DiVinE 3.0 - An Explicit-State Model Checker for Multithreaded C \& C ++ Programs. In: Sharygina, N., Veith, H. (eds.) CAV 2013. LNCS, vol. 8044, pp. 863-868. Springer, Heidelberg (2013)

11. Laarman, A., van de Pol, J., Weber, M.: Multi-Core LTSmin: Marrying Modularity and Scalability. In: Bobaru, M., Havelund, K., Holzmann, G.J., Joshi, R. (eds.) NFM 2011. LNCS, vol. 6617, pp. 506-511. Springer, Heidelberg (2011)

12. University of Oxford, Failures-Divergence Refinement-FDR 3 User Manual (2013), https://www.cs.ox.ac.uk/projects/fdr/manual/

13. University of Oxford, libcspm (2013), https://github.com/tomgr/libcspm

14. Reed, G.M., Roscoe, A.W.: A Timed Model for Communicating Sequential Processes. Theoretical Computer Science 58 (1988)

15. Armstrong, P., Lowe, G., Ouaknine, J., Roscoe, A.W.: Model checking Timed CSP. In: Proceedings of HOWARD (Festschrift for Howard Barringer) (2012)

16. Ouaknine, J.: Discrete Analysis of Continuous Behaviour in Real-Time Concurrent Systems. DPhil Thesis (2001)

17. Barringer, H., Kuiper, R., Pnueli, A.: A really abstract concurrent model and its temporal logic. In: Proceedings of the 13th ACM SIGACT-SIGPLAN Symposium on Principles of Programming Languages. ACM (1986)

18. Roscoe, A.W., Hopcroft, P.J.: Slow abstraction via priority. In: Liu, Z., Woodcock, J., Zhu, H. (eds.) Theories of Programming and Formal Methods. LNCS, vol. 8051, pp. 326-345. Springer, Heidelberg (2013)

19. Roscoe, A.W.: Model-Checking CSP. In: A Classical Mind: Essays in Honour of CAR Hoare (1994)

20. Goldsmith, M., Martin, J.: The parallelisation of FDR. In: Proceedings of the Workshop on Parallel and Distributed Model Checking (2002)

21. Leiserson, C.E., Schardl, T.B.: A work-efficient parallel breadth-first search algorithm (or how to cope with the nondeterminism of reducers). In: Proc. 22nd ACM Symposium on Parallelism in Algorithms and Architectures, SPAA 2010 (2010)

22. Korf, R.E., Schultze, P.: Large-scale parallel breadth-first search. In: Proc. 20th National Conference on Artificial Intelligence, vol. 3. AAAI (2005)

23. Holzmann, G.J.: Parallelizing the Spin Model Checker. In: Donaldson, A., Parker, D. (eds.) SPIN 2012. LNCS, vol. 7385, pp. 155-171. Springer, Heidelberg (2012)

24. Laarman, A., van de Pol, J., Weber, M.: Boosting multi-core reachability performance with shared hash tables. In: Formal Methods in Computer-Aided Design (2010) 